\title{
Broadband spectroscopy for characterization of tissue-like phantom optical properties
}

\author{
Ali SHAHIN ${ }^{1}$, Wesam BACHIR ${ }^{1, a}$ \\ ${ }^{I}$ Biomedical Photonics Laboratory, Higher Institute for Laser Research and Applications, Damascus University, \\ Damascus airport freeway, 11-111 Damascus, Syria \\ ${ }^{a}$ E-mail address: wesambachir002@gmail.com
}

(received 20 June 2017; accepted 25 October 2017)

\begin{abstract}
Optical phantoms are widely used for evaluating the performance of biomedical optical modalities, and hence, absorbing and scattering materials are required for the construction of optical phantoms. Towards that aim, new readily available and inexpensive black Ink (Parker) as a simulating absorber as well as Intralipid 20\% as a simulating scatterer are thoroughly investigated. Broadband Transmittance and Diffuse reflectance spectroscopic measurements were performed in the visible range $400-700 \mathrm{~nm}$. Optical properties of the phantom materials are determined. Analytical expressions for absorption and scattering coefficient related to the concentrations and wavelength of the Parker ink and Intralipid are also presented and discussed. The results show nonlinear trend in the absorption coefficient of Parker ink over the examined visible spectral range. Furthermore, Intralipid scattering coefficient variation across the mentioned spectral range shows a tissue-like scattering trend. The findings demonstrate the capability of the broadband transmission and diffuse reflectance for characterizing tissue-like phantom materials in the examined spectral range.
\end{abstract}

Key words: optical phantoms; Parker ink; Intralipid; diffuse reflectance spectroscopy.

\section{Introduction}

Recently, there has been enormous interest in optical methods and their medical applications since they have a distinct advantage of being inherently non-invasive [1]. Tissue simulating phantoms that mimic the optical properties of human and animal tissues are needed for the development of optical diagnostic systems and physical therapeutic interventions [2].

They can be utilized for testing and validation the performance of optical system designs. Since biological tissue is a turbid medium, optical phantom must have scattering and absorbing components [3].

Accordingly, understanding light-tissue interaction requires in-depth knowledge of optical coefficients, in particular absorption coefficient $\mu_{a}$, scattering coefficient $\mu_{s}$, and anisotropy factor $\mathrm{g}$. These coefficients mainly depend on the composition of tissue, and properties of incident light. In order to measure these coefficients, there are two strategies, direct and indirect methods. Indirect method calculations are based on measurement from reflectance, and transmittance spectra to deduce the optical coefficients [4].

On the other hand, different materials have been used for simulating absorption and scattering processes in biological tissues. However, liquid phantoms are among the most commonly used phantoms in biophotonic studies [5-7]. Liquid optical phantoms construction is simple and straightforward.
India ink, food dyes, and blood were used as components of phantom to mimic absorption coefficient of tissue [8]. However, previous studies on India ink have shown that it could not be considered as an absolute absorber. Madsen et al [9] investigated several batches of India ink samples from different brands and demonstrated that an India ink has small scattering component besides its absorption component, Nevertheless, all studies revealed that scattering effect of ink is very small compared with absorbing effect.

For scattering simulating materials, the most common scattering media used in tissue phantom manufacturing, are fat emulsions (Intralipid, Nutralipid, Liposyn) [10,11]. These products contain soybean oil, egg phospholipids, and glycerol and are the suspensions of roughly spherical fat droplets dispersed in water and whose optical properties could be predicted by Mie theory. Since these emulsions are produced for medical application and not as optical standards, their properties may vary with different batches and the measured optical properties may vary from one research group to another $[12,13]$. All prior research works, aimed to calculate the optical properties of fat emulsion, showed the variation of its albedo value, indicating they have an absorption effect.

The purpose of this study is to characterize the optical properties of new absorbing material, i.e. Parker ink and Intralipid $20 \%$ as a scattering constituent in liquid phantoms over a broadband wavelength range $400-650 \mathrm{~nm}$. Fiber based 
spectrophotometric transmittance set up, and diffuse reflectance spectroscopy for estimating the absorption and scattering coefficients of India ink and Intralipid 20\% respectively are presented.

\section{Materials and methods}

\section{Sample preparation}

Parker ink (Parker, Quink, England), was used as an absorbing medium and Intralipid 20\% (I141, Sigma Aldrich, Germany) as a scattering medium. Both Parker ink and Intralipid were diluted in distilled water to obtain concentrations from 0.01 to $0.1 \%$ for ink and from 0.01 to $0.07 \%$ for Intralipid $20 \%$ for transmittance measurement. The dilution process was intended to avoid the influence of multiple events i.e. scattering in Intralipid and absorption in Parker ink.

All samples were kept in a plastic cuvette that has a $1 \mathrm{~mm}$ thickness $1 \mathrm{~cm}$ path length. For diffuse reflectance measurement, samples of Intralipid were kept in a $500 \mathrm{ml}$ glass beakers with concentrations of $0.04,0.06,0.08,0.1,0.12$, and $0.2 \%$. The sample volume was enlarged to avoid the leaking of light from the sides of beaker and to validate the approximation of semi-infinite medium.

\section{Spectrophotometric transmission spectroscopy}

A broad band, fiber based spectrophotometric transmission setup was arranged. Figure 1 shows a schematic drawing of the experimental set up. It consists of broadband halogentungsten light source (HL-2000-HP-FHSA, Ocean Optics Inc. FL), a fiber coupled cuvette holder with two collimating lenses (CUV-ATT-DA, Avantes Inc., Netherlands), and a USB portable spectrometer (USB4000 FL, Ocean Optics Inc.). The attenuation coefficient of Parker ink and Intralipid samples can be measured within a broad wavelength range from $400-$ $700 \mathrm{~nm}$.

With this collimated transmission setup, the attenuation coefficient of a sample can be measured. The sample was illuminated with a collimated white light source. Thus, the transmission intensity may be approximated by Beer Lambert Law (Equations 1-2).

$I_{z}(\lambda)=I_{0}(\lambda) \cdot e^{-\mu_{t}(\lambda) \cdot z \cdot c}$

$\frac{I_{Z}(\lambda)}{I_{0}(\lambda)}=T$

Where $I_{z}(\lambda)$ is the intensity of light passed through the studied sample, $I_{0}(\lambda)$ is the reference intensity, $\mathrm{z}$ is the optical path length (thickness of cuvette), and $c$ is the concentration. The distance between the sample and the detector was made as large as possible in order to detect only non-scattered photons. Then, the measured attenuation coefficient of high scattering samples $\mu_{t}(\lambda)$ equals the scattering coefficient $\mu_{s}(\lambda)$ and this may apply to Intralipid samples. For absorbing samples, the measured attenuation coefficient equals the absorption coefficient $\mu_{a}(\lambda)$.

\section{Diffuse reflectance spectroscopy}

A schematic description of the diffuse reflectance spectroscopy setup is shown in Figure 2. Light from a tungsten halogen lamp (Ocean Optics Inc., Dunedin, Florida, USA, HL-20000HPFHSA) was delivered to the sample of interest through a central optical fiber of a two-leg fiber optic probe (R600-7VIS-125F, Ocean Optics Inc., Dunedin, Florida, USA) with a core diameter of $600 \mu \mathrm{m}$ and a numerical aperture of 0.22 .

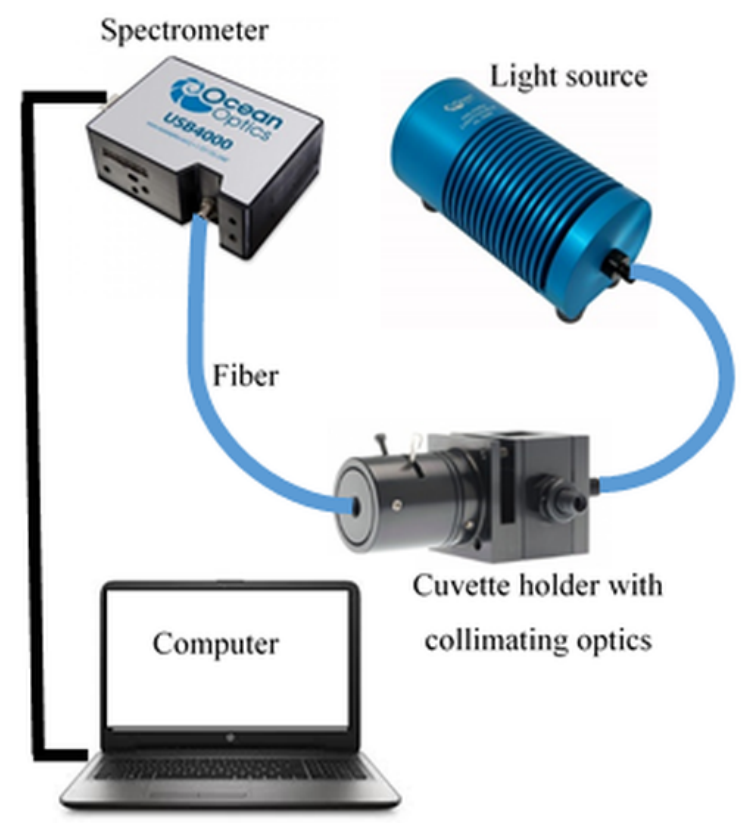

Figure 1. Experimental measurement setup for transmission spectroscopy.

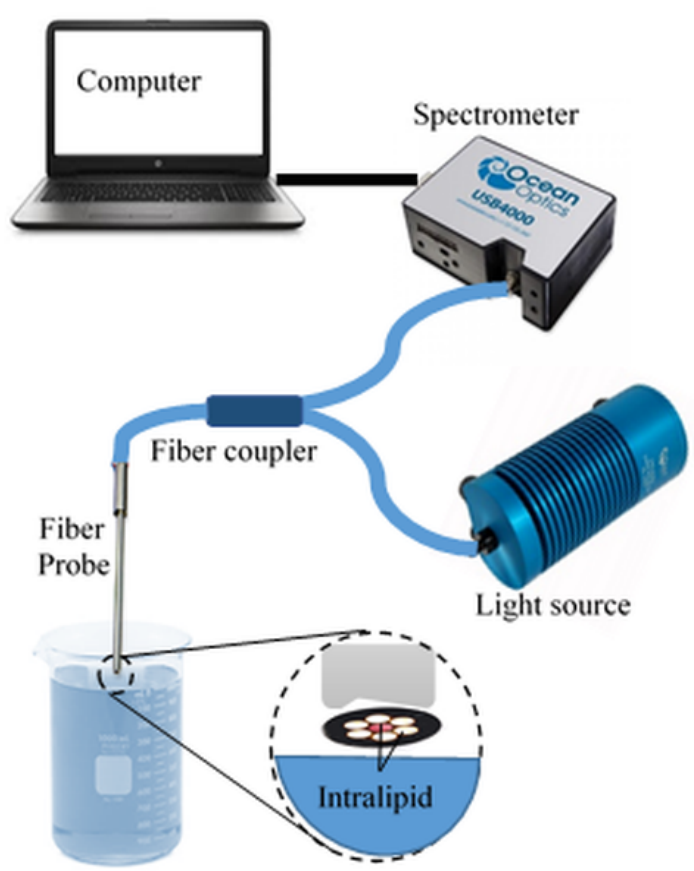

Figure 2. Schematic drawing of the setup arrangement for fiber based diffuse reflectance spectroscopy. 
A back-scattered light emanated from the sample is guided through the second leg of the optical fiber probe and coupled to a miniature fiber optic spectrometer (Ocean Optics Inc., Dunedin, Florida, USA). The fiber optic spectrometer is connected to a computer for data acquisition and further analysis. The spectrometer has a $600-$ lines $/ \mathrm{mm}, 200-\mu \mathrm{m}$ slit, $500-\mathrm{nm}$ blazed grating for operation in the 345 to $1080 \mathrm{~nm}$ wavelength range.

The USB-based spectrometer is equipped with a 3648element linear silicon CCD array detector. The spectrum was acquired in the 400 to $650 \mathrm{~nm}$ spectral ranges with the help of the SpectraSuite software (Ocean Optics Inc., USA, version 2011). Dark current and background measurements were taken at the same setting before each recording.

Diffuse reflection spectrum was measured in a semi-infinite medium and post processed in order to estimate the reduced scattering coefficient $\mu_{s}^{\prime}(\lambda)$ based on Equation 3.

$\mu_{s}^{\prime}(\lambda)=\mu_{s}(\lambda) \cdot(1-g)$

Where $\mu_{s}(\lambda)$ is the scattering coefficient and $\mathrm{g}$ is the anisotropy factor. In this way, the diffuse reflectance of the sample was measured by diffuse reflectance spectroscopy. Then, the measured diffuse reflectance was used to estimate the reduced scattering coefficient of Intralipid using the following formula:

$R_{\infty}=\frac{a^{\prime}}{1+2 \cdot K\left(1-a^{\prime}\right)+\left(1+\frac{2 \cdot K}{3}\right) \cdot \sqrt{3 \cdot\left(1-a^{\prime}\right)}}$

Eq. 4

Where $\mathrm{R}_{\infty}$ is the measured diffuse reflectance, and $a^{\prime}$ is the reduced albedo expressed as follows:

$a^{\prime}=\mu_{s}^{\prime} /\left(\mu_{s}^{\prime}+\mu_{a}\right)$

$\mathrm{K}=(1+\mathrm{r}) /(1-\mathrm{r})$

Where $r$ is the internal diffuse reflectance that can be calculated from the following expression:

$r=-1.44 n_{\text {rel }}^{-2}+0.71 n_{\text {rel }}^{-1}+0.668+0.0636 n_{\text {rel }}$

and the relative index of refraction is given by:

$n_{\text {rel }}=n_{\text {intralipid }} / n_{\text {air }}$

This equation is more accurate for samples with high scattering component compared to the absorption component, therefore this method may be used for absolute scattering medium in order to estimate the albedo value for Intralipid $20 \%$ samples over broadband range. Therefore, white light from a halogen light source was used to illuminate perpendicularly the surface of liquid sample by means of bifurcated fiber probe as shown in Figure 2. The distance between the tip of the probe and the surface of aqueous solution was set to nearly $5^{\circ}$ of collection angle to minimize the undesired specular reflection.

\section{Results and discussion}

Figure 3a shows the absorption coefficient of Parker ink as a function of wavelength. It can be seen from the figure that the variation of absorption coefficient is nonlinear with wavelength.

Equation 9 was found to provide the fit to the absorption coefficient measurements $\left(\mathrm{R}^{2}=0.977\right)$ as shown in Figure 3b, with fitting coefficients values listed in Table 1. Curve fitting procedure was carried out using Curve fitting toolbox software in Matlab 2016 (Mathworks Inc., USA)

$\mu_{a}(\lambda)=A_{1} e^{\left(-\left(\frac{\lambda-B_{1}}{C_{1}}\right)\right)^{2}}+A_{2} 2 e^{\left(-\left(\frac{\lambda-B_{2}}{C_{2}}\right)\right)^{2}}$

To the best of our knowledge, Parker ink has not been investigated for optical phantom development. With respect to Parker ink spectrophotometric measurements, it was found, contrary to expectations, a significant difference between Parker ink absorption spectrum and the absorption spectrum of India Ink reported in the literature. However, this difference might be attributed to the different chemical composition of Parker ink. However, absorption spectrum Parker ink in the visible range of wavelengths might be divided into sub regions where the trend is linear with wavelength.

Also, the results observed from the current experimental work reveals that there has been a steep decline in the absorption coefficient of Parker ink from around $600 \mathrm{~nm}$ upwards similar to the behavior of India ink.

The relationship between absorption coefficient of Parker ink and its concentration $\mathrm{C}$ has also been experimentally examined. Figure 3c shows the variation in absorption coefficient of Parker ink with increased concentration. These measurements, taken at $600 \mathrm{~nm}$, were fitted linearly with $\mathrm{R}^{2}$ of 0.994 according to Equation 10.

$\mu_{a}(\lambda)=195.9 C+0.02$

Eq. 10

For Intralipid $20 \%$ material, transmittance spectra of Intralipid $20 \%$ are shown in Figure 4a. The transmittance of Intralipid $20 \%$ was found to be directly proportional to the increased wavelength. Diffuse reflectance spectroscopy makes it possible to estimate the reduced scattering coefficient. As a result, the scattering coefficient for given values of anisotropy factor can be determined. Therefore, the values of anisotropy factor were taken from the literature [10], and then scattering coefficient values of Intralipid $20 \%$ and their corresponding albedo values at some specific wavelengths $405 \mathrm{~nm}, 543 \mathrm{~nm}$, $625 \mathrm{~nm}, 632 \mathrm{~nm}$, and $800 \mathrm{~nm}$ were calculated.

Our measurements showed that Intralipid $20 \%$ can be considered as an ideal scattering medium at $405 \mathrm{~nm}$, because the value of albedo at this wavelength $405 \mathrm{~nm}$ was found to equal one. 

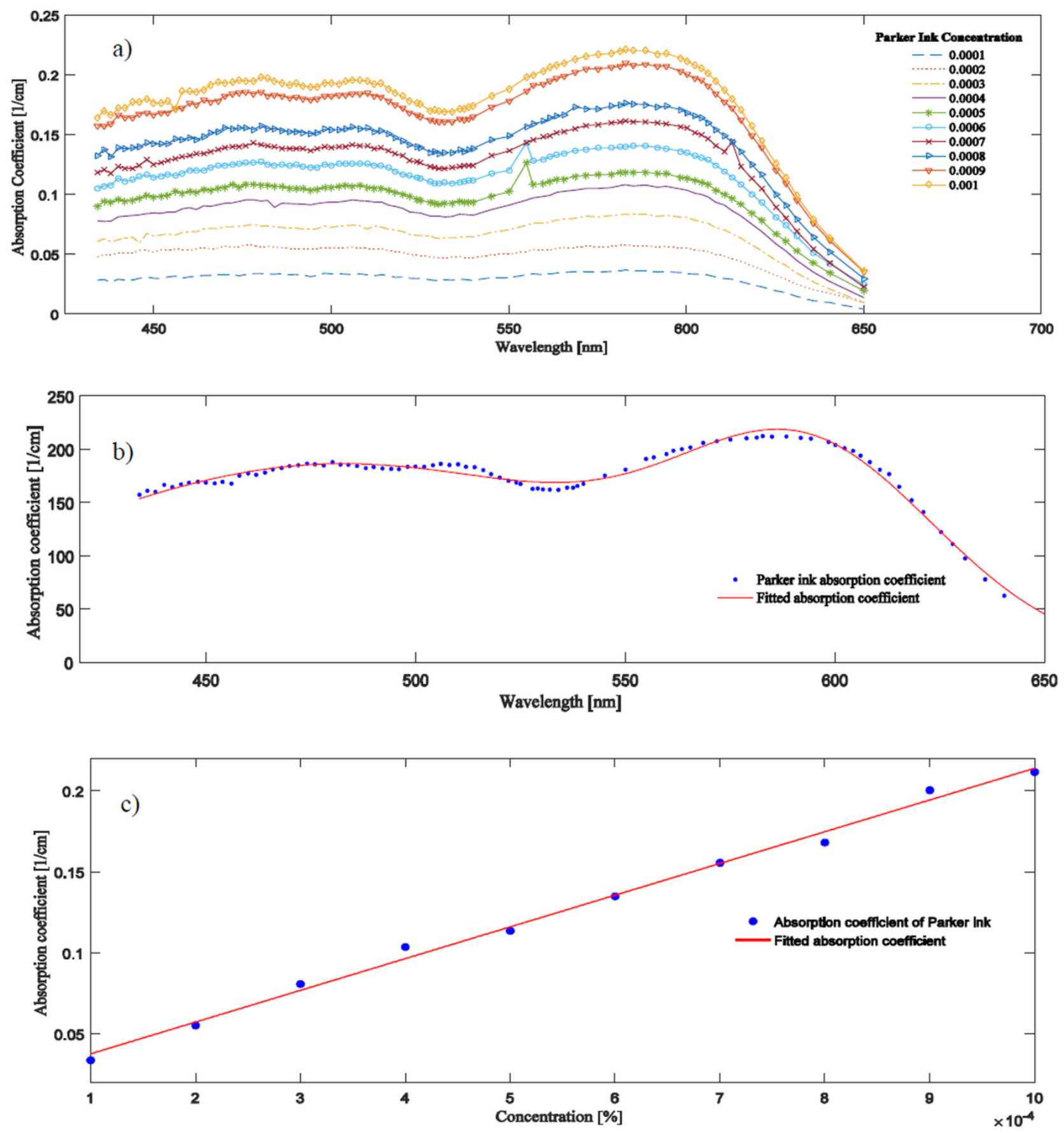

Figure 3. Spectrophotometric measurement of Parker ink a) the absorption coefficient of Parker ink as a function of wavelength for a variety of concentrations. b) fitted model for absorption coefficient of Parker ink as a function of wavelength. c) fitted model for absorption coefficient of Parker ink as a function of concentration at $600 \mathrm{~nm}$.

Table 1. Fitting coefficients for absorption coefficient of Parker ink as a function of wavelength.

\begin{tabular}{ccccccc}
\hline \hline Fitting coefficient & $\mathbf{A}_{\mathbf{1}}$ & $\mathbf{B}_{\mathbf{1}}$ & $\mathbf{C}_{\mathbf{1}}$ & $\mathbf{A}_{\mathbf{2}}$ & $\mathbf{B}_{\mathbf{2}}$ & $\mathbf{C}_{\mathbf{2}}$ \\
\hline Value & 149 & 1.095 & 0.7148 & 186.3 & -0.7851 & 1.829 \\
\hline \hline
\end{tabular}


a)

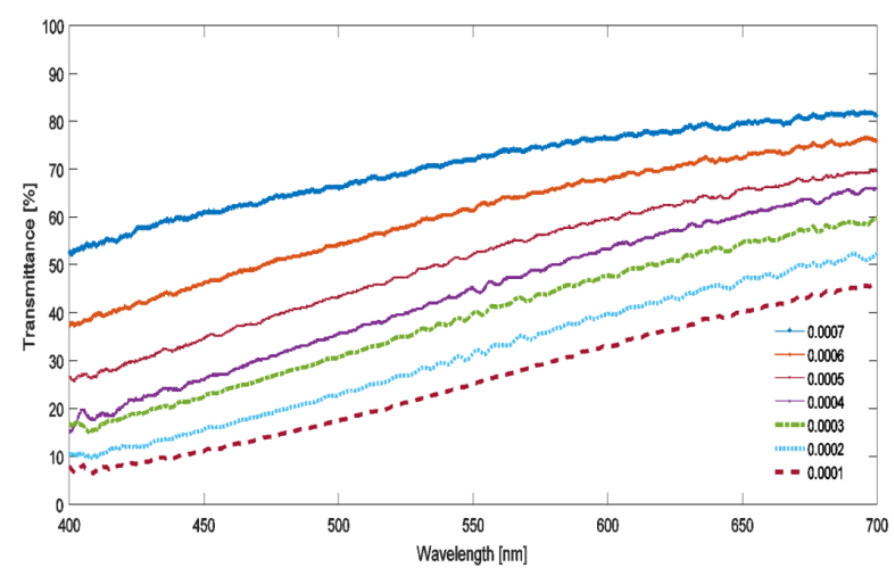

c)

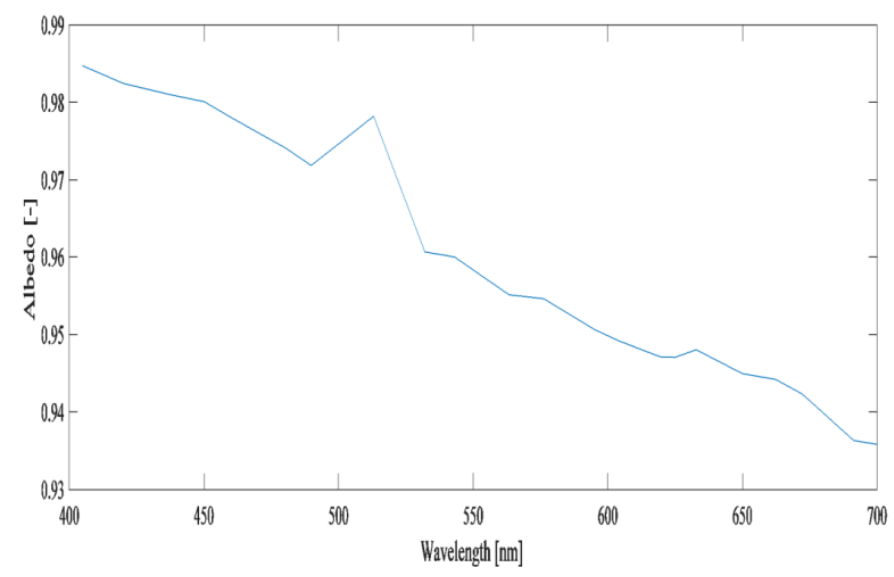

b)
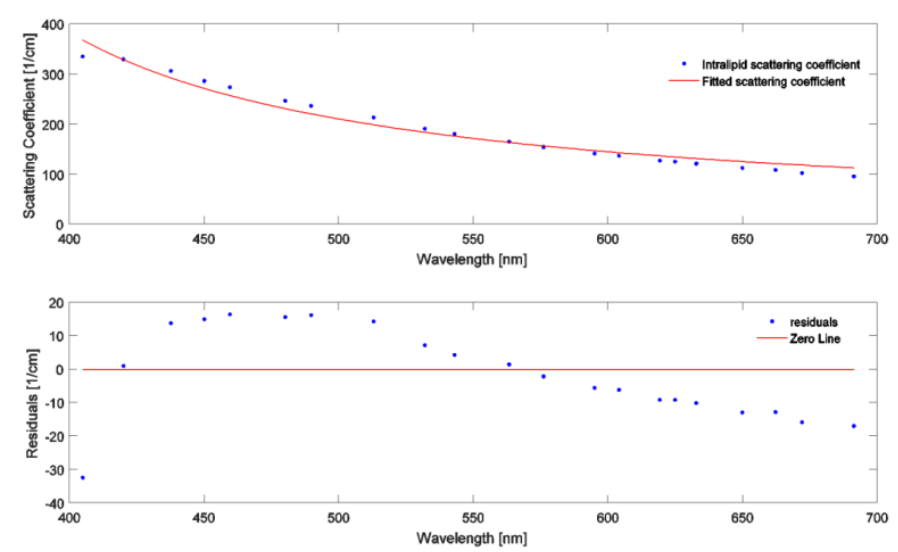

d)

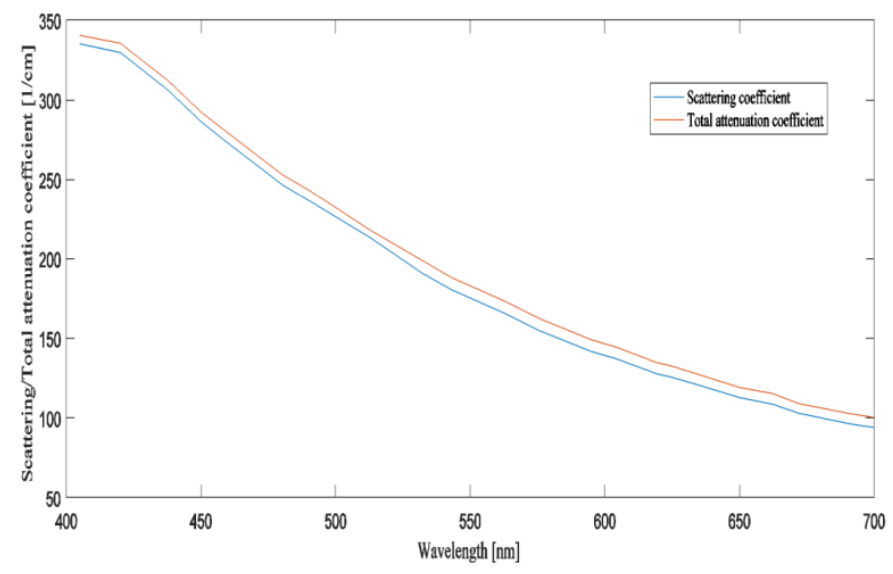

Figure 4. a) Transmittance spectra of Intralipid $20 \%$ within the visible range. b) Fitted model for the scattering coefficient of Intralipid $20 \%$. c) Intralipid $20 \%$ albedo as function of wavelength. d) Total attenuation and scattering coefficients of Intralipid as a function of wavelength.

In addition, the results observed from diffuse reflectance spectroscopy measurements were found to be in line with those of prior studies. That is, the estimated of scattering coefficient of Intralipid 20\% showed declined trend with increased wavelength as can be observed from Figure $4 \mathbf{b}$. Consequently, a fitted analytical model $\left(\mathrm{R}^{2}=0.97\right)$ for the scattering coefficient as a function of wavelength was attained as follows:

$\mu_{s}(\lambda)=4.679 \mathrm{e}^{4} /(\lambda-277.7)$

The decline of Intralipid $20 \%$ scattering was found to be inversely proportional to the wavelength. This is in consistence with scattering of biological tissue based on Mie theory [15].

Additionally, the calculated albedo of Intralipid 20\% is plotted in Figure 4c. Figure 4d compares the total attenuation coefficient from transmission spectroscopy measurement and the estimated scattering coefficient of Intralipid $20 \%$.

Nonetheless, diffuse reflectance spectroscopy is a simple and rapid, indirect method to quantify the scattering coefficient of Intralipid $20 \%$. It is noteworthy to say that the abovementioned estimation of scattering coefficient of Intralipid does not require the value of absorption coefficient unlike previous works on Intralipid scattering measurements provided in the literature.

\section{Conclusion}

In summary, the results given in this paper was an attempt to investigate the optical properties of Parker ink as an absorbing material. It can be concluded that Parker ink can be used as an absorbing constituent in optical phantom. Yet, one should take into account the nonlinear relation between absorption coefficient of Parker ink and wavelength unlike India ink.

Another important conclusion is that scattering coefficient of Intralipid $20 \%$ can be assessed by diffuse reflectance spectroscopy. The optical characterization of these main phantom materials provide a guiding approach when constructing liquid optical phantoms for validating theoretical and experimental biophotonic instrumentation. Diffuse reflectance spectroscopy, which is already considered as a diagnostic tool in medicine [16], can also be utilized as a testing method to estimate the scattering coefficient of the Intralipid $20 \%$. As a result, this may well provide a course tuning of the optical properties and concentrations of these materials when constructing tissue-like liquid phantoms. 


\section{References}

[1] Tromberg BJ, Anderson RR, Birngruber RR, et al., Biomedical optics centers: forty years of multidisciplinary clinical translation for improving human health. J Biomed Opt. 2016;21(12):pp. 23-35.

[2] Pogue BW, Patterson MS. Review of tissue simulating phantoms for optical spectroscopy, imaging and dosimetry. J Biomed Opt. 2006;11(4):041102-16.

[3] Hwang J, Ramella-Roman CJ, Nordstrom R. Introduction: Feature Issue on Phantoms for the Performance Evaluation and Validation of Optical Medical Imaging Devices. Biomed Opt Express. 2012;3(6):1399-1403.

[4] Flock ST, Jacques LS, Wilson CB, et al. Optical properties of Intralipid: a phantom medium for light propagation studies. Lasers Surg Med. 1992;12(5):510-519.

[5] Moffitt T, Chen CY, Prahl AS. Preparation and characterization of polyurethane optical phantoms. J Biomed Opt. 2006;11(4): 041103-10.

[6] Lamouche G, Kennedy FB, Kennedy MK, et al. Review of tissue simulating phantoms with controllable optical, mechanical and structural properties for use in optical coherence tomography. Biomed Opt Express. 2012;3(6):1381-1398.

[7] Chang RC, Johnson P, Stafford CM, Hwang J. Fabrication and characterization of a multilayered optical tissue model with embedded scattering microspheres in polymeric materials. Biomed Opt Express. 2012;3(6):1326-1339.

[8] Ninni DP, Martelli F, Zaccanti G. The use of India ink in tissue-simulating phantoms. Opt Express. 2010;18(26):26854-65.

[9] Madsen SJ, Patterson MS, Wilson BC. The use of India ink as an optical absorber in tissue-simulating phantoms. Phys Med Biol. 1992;37(4):985-993.

[10] Michels R, Foschum F, Kienle A. Optical properties of fat emulsions. Opt Express. 2008;16(8):5907-5925.

[11] van Staveren HJ, Moes CJ, van Marle J, et al. Light scattering in Intralipid-10\% in the wavelength range of 400-1100 nm. Appl Opt. 1991:30(31): 4507-4514.

[12] Ninni PD, Martelli F, Zaccanti G. Intralipid: towards a diffusive reference standard for optical tissue phantoms. Phys Med Biol. 2011;56(2):N21-N28.

[13] Spinelli., Martelli F, Farina A, et al. Calibration of scattering and absorption properties of a liquid diffusive medium at NIR wavelengths. Time-resolved method. Opt Express. 2007;15(11): 6589-6604.

[14] Welch AJ, van Gemert MJC. Optical-Thermal Response of Laser-Irradiated Tissue. Springer, 2011, pp. 279-286.

[15] Jacques SL. Optical properties of biological tissues: a review. Phys Med Biol. 2013;58(11):R37-R61.

[16] Hafez R, Hamadah O, Bachir W. Mapping of healthy oral mucosal tissue using diffuse reflectance spectroscopy: ratiometric-based total hemoglobin comparative study. Lasers Med Sci. 2015;30(8):2135-2141. 